\title{
Study of the multiple-profile control system to enhance oil recovery after polymer flooding
}

\author{
Q. H. Feng $\cdot$ X. C. Chen $\cdot$ M. D. Sun
}

Received: 10 July 2011 / Accepted: 7 July 2012 / Published online: 26 July 2012

(C) The Author(s) 2012. This article is published with open access at Springerlink.com

\begin{abstract}
There is still half oil remaining in the reservoir after polymer flooding. This paper has carried out the laboratory studies on multi-profile control and displacement system to enhance oil recovery after polymer flooding. First of all, the multiple system comprising a gel particle, cross-linking agent and high effective surfactant, is screened according to specific criteria. Then the profile control experiments on binary and ternary system after polymer flooding are separately carried out to verify the potential of enhanced oil recovery. The results show that the multiple injection system not only blocks the high permeable layers and channels, but also makes full use of medium and low permeability layers, finally improving the whole sweep efficiency. Owing to the surfactant injection, the displacement efficiency is simultaneously improved to some extent, and thus greatly improves the oil recovery after polymer flooding.
\end{abstract}

Keywords Post polymer flood - Gel particles . Cross-linking agent $\cdot$ Surfactant $\cdot$ Multi-profile control and displacement system $\cdot$ Enhanced oil recovery (EOR)

\section{Introduction}

Field experience has proven that polymer flooding can be an effective means to improve oil recovery after water

Q. H. Feng · X. C. Chen $(\bowtie)$

China University of Petroleum (East China), Qingdao,

Shandong, China

e-mail: mangix2010@gmail.com

M. D. Sun

Shengli Oil Field of SINOPIC, Dongying, Shandong, China flooding. Polymer flooding plays the role by adding watersoluble polymer in injection water to increase the injection viscosity (Needham and Doe 1987; Kaminsky et al. 2007). The reason why polymer flooding can enhance oil recovery relies on that it can improve areal and vertical sweep efficiency as well as providing mobility control (Gogarty 1978; Davison and Mentzer 1982; Wang et al. 2002; Wang and Liu 2004). As an important enhanced oil recovery technique, polymer flooding is currently widely used in China's onshore oilfields. For example, the world's largest polymer flooding was implemented at Daqing Oilfield, beginning in 1996. By 2007, $22.3 \%$ of total production from the Daqing Oil Field was attributed to polymer flooding (Dong et al. 2008). Polymer flooding, which is also widely used in offshore oilfields in China, has become an important enhanced oil recovery (EOR) technique recently for offshore oilfields development (Ming et al. 2006; Wei et al. 2007; 2008).

Although polymer flooding can boost the oil recovery to over $50 \%$, which is about $10 \%$ higher than water flooding (Jiecheng et al. 2010), there is still half oil remaining in the reservoir. The effect of polymer flooding is limited due to the following reasons: Firstly, the heterogeneity is aggravated during the polymer flooding process, in which the water and polymer fingering phenomena happens along thief zones and high permeability streak resulting in low sweep efficiency. Secondly, the polymer displacement formula is not able to reduce the interfacial tension, so the displacement efficiency is low. In those means, how to improve the oil recovery after polymer flooding can take both the sweep efficiency and displacement efficiency into account (Jishui and Yiqiang 2002; Ting and Xiusheng 2004; Stoll et al. 2010).

The key to enhance oil recovery after polymer flooding is to improve the sweep efficiency of injected water. The 
Table 1 Degree of reserve recovery for gel particle profile control

\begin{tabular}{lllllll}
\hline $\begin{array}{l}\text { Gel particle } \\
\text { type }\end{array}$ & $\begin{array}{l}\text { Pore } \\
\text { volume }(\mathrm{ml})\end{array}$ & $\begin{array}{l}\text { RF of water } \\
\text { flooding }(\%)\end{array}$ & $\begin{array}{l}\text { RF of polymer } \\
\text { flooding }(\%)\end{array}$ & $\begin{array}{l}\text { RF of profile } \\
\text { control }(\%)\end{array}$ & $\begin{array}{l}\text { Increased RF after polymer } \\
\text { flooding }(\%)\end{array}$ & $\begin{array}{l}\text { Increased RF after profile } \\
\text { control }(\%)\end{array}$ \\
\hline Cation & 119.23 & 34.81 & 54.05 & 64.24 & 19.24 & 10.19 \\
Cation & 148.39 & 35.12 & 47.71 & 58.74 & 12.59 & 11.03 \\
Anion & 107.70 & 34.69 & 52.65 & 54.69 & 17.96 & 2.04 \\
Anion & 139.50 & 33.81 & 48.09 & 50.94 & 14.28 & 2.85 \\
\hline
\end{tabular}

current most used method is polymer gel profile control technology such as preformed gel particles (Coste et al. 2000; Baojun et al. 2007a, b), which can divert the fluid to flow across the upswept area. In consideration of the abundant remaining polymer in the reservoir after polymer flooding, the cross-linking agent can be injected to react with the residual polymer to form the gel, which can block high permeability zone and activate the medium-low permeability zone. As a result, the sweep efficiency of water flooding and oil recovery can be enhanced (Yefei et al. 2006; Dai et al. 2010). The efficient surfactant can also be injected to improve the low permeability layer displacement efficiency and bring down the follow-up water injection pressure. The above three injection agents make up the multiple-profile control system, which not only enlarges the follow-up water flooding sweep efficiency but also increases the displacement efficiency to some extent (Abdo and Chung 1984). The high, medium and low permeability layers are all made full use of, thus the oil recovery after polymer flooding goes up a lot.

Based on previous research, the multiple-profile control system components are screened separately. The major focus is the effect of the system in core flooding after polymer flooding, including the water cut decline, the oil recovery improvement and the injection pressure reduction. The potential of enhancing oil recovery of the multipleprofile control system can be evaluated from the experiment results analysis.

\section{Multiple-profile control system}

\section{Gel particle type screening}

The layered heterogeneous sand pack cores are used in screening experiment. The core size is $4.5 \times 4.5 \times 30.0 \mathrm{~mm}$ and the permeability max-min ratio is 8 (The terminology describes the degree of permeability heterogeneity, and is defined as the ratio of the max layer permeability and min layer permeability). The cation gel particle and the anion gel particle are going to be screened by the relationship between the injected PV and the recovery factor of the core profile control experiment (Shaodong and Xiangguo 2002). In both profile control experiments, the water flooding is the first displacement stage, and the polymer flooding is the second displacement stage. The gel particle solution is injected after the polymer flooding. The results of the core flooding experiment are shown in Table 1.

The results show that the recovery factors of water and polymer flooding for cation and anion are nearly same, but the recovery factor of cationic gel particle is eight percents higher than anion after profile control displacement stage. The reason lies in the electrostatic adsorption reaction between the cationic gel particle and the anion residual polymer on the rock surface, which larges the grain diameter of cationic gel particle. Therefore, the blocking effect of cationic gel is better than anion gel particle.

Cross-linking agent concentration screening

The screening experiment is also adopting the layered heterogeneous sand pack core, the displacement experiments of different concentration cross-linking agent are carried out after polymer flooding (Dejun and Hui 2002). The recovery factor of different scenes are shown in Table 2.

The results show that the recovery factor after polymer flooding is basically same for the heterogeneous cores with same permeability max-min ratio. The cross-linking agent can considerably improve the recovery factor after polymer flooding. The concentration $100 \mathrm{mg} / \mathrm{L}$ obtained the highest rate of EOR effect.

\section{High effective surfactant concentration screening}

The optimal surfactant concentration can be screened according to the interfacial tension of different surfactant concentration solution (XiaoQuan and Xijing 2004; Monika et al. 2011). The interfacial tension variation can be seen from Fig. 1. When the concentration is $0.2 \%$, the interfacial tension achieves the minimum value. So the $0.2 \%$ will be used as the optimal concentration.

In order to verify the depressurization effects of the $0.2 \%$ concentration surfactant, the low permeability core displacement experiment is conducted. The injection pressure data with injected PV is recorded. The results of 0.1 and $0.2 \%$ are shown in Fig. 2. 
Table 2 The degrees of reserve recovery of different concentration cross-linker

\begin{tabular}{lllcll}
\hline Core size & $\begin{array}{l}\text { Permeability } \\
\text { max-min ratio }\end{array}$ & $\begin{array}{l}\text { Pore volume } \\
(\mathrm{mL})\end{array}$ & $\begin{array}{l}\text { Cross-linker concentration } \\
(\mathrm{mg} / \mathrm{L})\end{array}$ & $\begin{array}{l}\text { RF after polymer } \\
\text { flooding }(\%)\end{array}$ & $\begin{array}{l}\text { RF after cross-linker } \\
\text { injection }(\%)\end{array}$ \\
\hline $30 \times 4.50 \times 4.50$ & 3 & 147.09 & 0 & 11.92 & 0 \\
$30 \times 4.50 \times 4.50$ & 3 & 152 & 50 & 10.64 & 5.5 \\
$30 \times 4.50 \times 4.50$ & 3 & 149.56 & 100 & 12.25 & 7.30 \\
$30 \times 4.50 \times 4.50$ & 5 & 150.01 & 0 & 17.85 & 0 \\
$30 \times 4.50 \times 4.50$ & 5 & 155.39 & 50 & 19.9 & 4.03 \\
$30 \times 4.50 \times 4.50$ & 5 & 158 & 100 & 16.62 & 5.7 \\
\hline
\end{tabular}

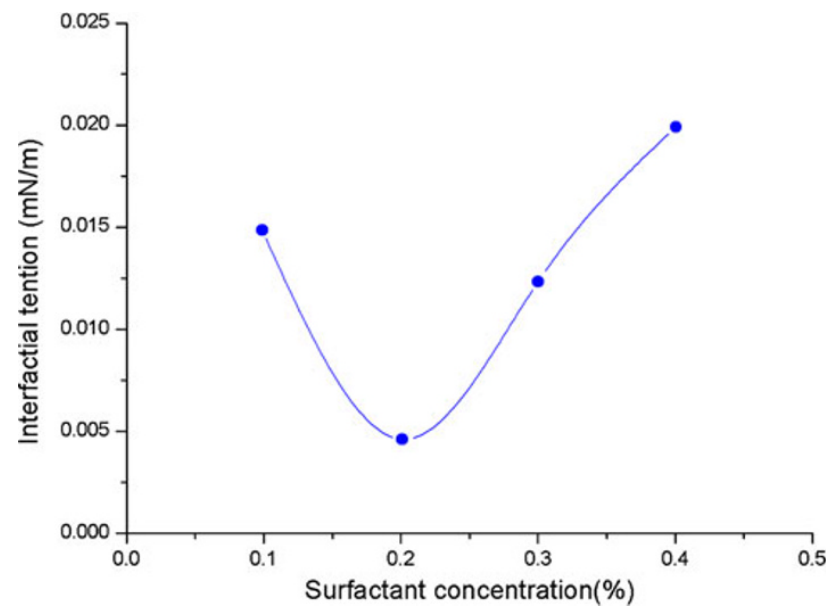

Fig. 1 The interfacial tension force curve of vary concentration oil displacing agents

It can be seen that the pressure in water and polymer flooding stage is almost the same, when it turns to surfactant flooding, the pressure begins to dramatically decline and the pressure continues to reduce at the following water flooding stage. This phenomenon certifies that the surfactant system can bring down the injection pressure by reducing the water-oil interfacial tension and residual oil saturation to raise the water phase relative permeability.

\section{Core flooding experiment}

Experiment equipment

\section{Material}

1. Reinjection water from a oil production plant in Daqing Oilfield, the salinity is $2841.2 \mathrm{mg} / \mathrm{L}$, the injection water belongs to bicarbonate-sodium type water and the impurities are removed using $0.2 \mu \mathrm{m}$ polycarbonate membrane filtration;

2. Cross-linking agent for the field application in Daqing Oilfield with $67 \%$ trivalent chromium ion and blackish green color;

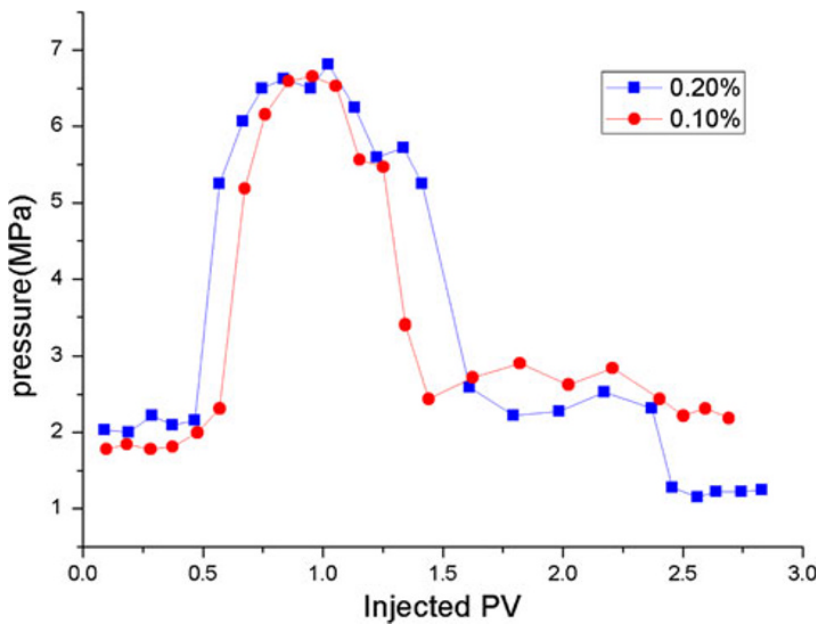

Fig. 2 Injection pressure variation curve of vary concentration oil displacing agents

3. Polymer with the molecular weight $16-19$ million and the hydrolyzing degree is $20 \%$;

4. Cationic gel particles used in Daqing Oilfield;

5. Efficient surfactant (anion sulfonic acid salt) with $50 \%$ effective content and the relative molecular weight is 420 ;

6. Oil mixture of dehydrated crude oil and jet fuel (the viscosity of crude oil is $9.8 \mathrm{mPa} \mathrm{s}$ at $45^{\circ} \mathrm{C}$, and the density is $0.925 \mathrm{~g} / \mathrm{cm}^{3}$ );

7. Artificial quartz sandstone core.

Instruments

1. Particle mixing device;

2. High pressure displacement pump: velocity range is $0.01-200 \mathrm{~mL} / \mathrm{min}$, working pressure is not less than $30 \mathrm{MPa}$;

3. Annular pump: working pressure is not less than $20 \mathrm{MPa}$; core holder: core sample size is $4.5 \times$ $4.5 \mathrm{~cm} \times 30 \mathrm{~cm}$ and the working pressure is not less than $16 \mathrm{MPa}$; 
Fig. 3 Flow chart of core flooding experiment

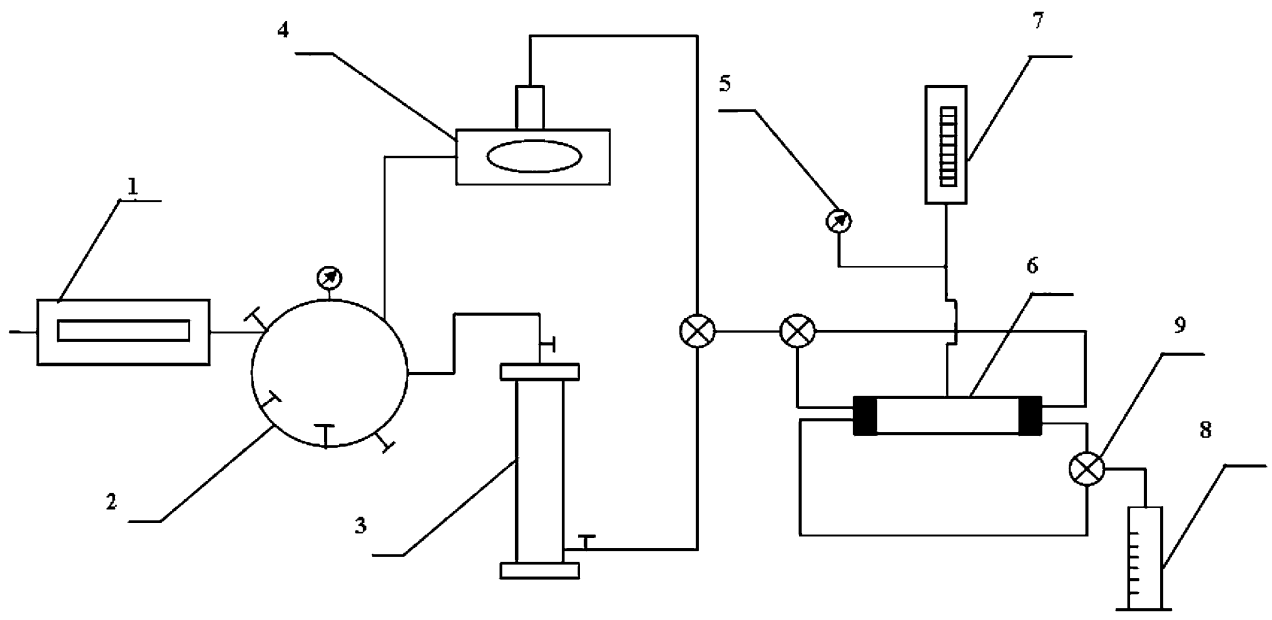

1- gas cylinder or high pressure displacement pump; 2-six-way valve or multiport valve; 3-high pressure intermediate container;4-particle agitator; 5-pressure gauge; 6-core holder; 7-annular pump; 8-gauge pipe or flow meter; 9- Three Ball valves
4. Manometer: Electronic pressure gauge, the measuring range is selected according to the experiment requirement; multi-pass set;

5. Graduated cylinder: Certificated graduated cylinder, the range is $20-50 \mathrm{~mL}$;

6. Electronic balance: sensibility reciprocal $10 \mathrm{mg}$.

The flow chart of the experiment is illustrated as Fig. 3.

\section{Experiment procedure}

Binary injection system profile control experiment

1. Vacuumize the core and saturate the core with water;

2. Saturate the core with oil;

3. Inject water until the water cut reaches $98 \%$;

4. Inject $0.7 \mathrm{PV}$ polymer solution with the concentration $1,000 \mathrm{mg} / \mathrm{L}$;

5. Inject $0.3 \mathrm{PV}$ water;

6. Inject $1 \mathrm{PV}$ mixture solution with $100 \mathrm{mg} / \mathrm{L}$ crosslinking agent and $2,000 \mathrm{mg} / \mathrm{L}$ cationic gel particles;

7. Keep for 2 days at $45{ }^{\circ} \mathrm{C}$ constant temperature;

8. Inject water until the water cut reaches $98 \%$.

Multiple injection system profile control experiment

1. Vacuumize the core and saturate the core with water;

2. Saturate the core with oil;

3. Inject water until the water cut reaches $98 \%$;

4. Inject $0.7 \mathrm{PV}$ polymer solution with the concentration $1,000 \mathrm{mg} / \mathrm{L}$;

5. Inject $0.3 \mathrm{PV}$ water;

6. Inject $0.3 \mathrm{PV} 2,000 \mathrm{mg} / \mathrm{L}$ cationic gel particles solution;

7. Inject $0.2 \mathrm{PV} 100 \mathrm{mg} / \mathrm{L}$ cross-linking agent solution;

8. Inject $0.2 \mathrm{PV} 0.2 \%$ surfactant solution;
9. Keep for 2 days at $45{ }^{\circ} \mathrm{C}$ constant temperature;

10. Inject water until the water cut reaches $98 \%$.

\section{Experimental results and analysis}

The Binary injection system profile control experiment

\section{Profile control effect}

The breakthrough pressure of different displacement ways is recorded in the core flooding process and the core permeability is measured after binary system injection. The blocking ratio (BR) and residual resistance factor (RRF) are obtained by calculation which is illustrated in Table 3 .

The BR is defined as following:

$B R=1-\frac{K_{f}}{K_{i}}$

Where, the subscript " $i$ " indicates the initial core permeability and the subscript " $f$ " indicates the final core permeability after profile control treatment.

The residual resistance factor is defined as following:

$\operatorname{FRR}=\frac{K_{\mathrm{rw}}}{K_{\mathrm{rp}}}$

where $K_{\mathrm{rw}}$ is the initial water phase relative permeability and $K_{\mathrm{rp}}$ is the water relative permeability after profile control treatment.

It can be seen that the breakthrough pressure of water flooding after binary system injection increased significantly. This phenomenon is created by the blocking effect of binary system. The grain diameter of gel particle is 
Table 3 The result of breakthrough pressure and RRF of injecting binary system

\begin{tabular}{llllllccc}
\hline NO & $\begin{array}{l}\text { Core perm. } \\
(\mathrm{mD})\end{array}$ & $\begin{array}{l}\text { Water flooding } \\
(\mathrm{MPa})\end{array}$ & $\begin{array}{l}\text { Polymer flooding } \\
(\mathrm{MPa})\end{array}$ & $\begin{array}{l}\text { Water after binary } \\
\text { system }(\mathrm{MPa})\end{array}$ & $\begin{array}{l}\text { Perm. after 4 PV } \\
\text { water injection }(\mathrm{mD})\end{array}$ & $\begin{array}{l}\text { RRF } \\
\text { BR }(\%)\end{array}$ & $\begin{array}{l}\text { Gel concentration } \\
(\mathrm{mg} / \mathrm{L})\end{array}$ \\
\hline 1 & 242 & 0.06 & 0.63 & 3.81 & 5.5 & 63.5 & 97.7 & 1,000 \\
2 & 735 & 0.021 & 0.192 & 3.63 & 4.6 & 372 & 99.3 & 1,000 \\
3 & 839 & 0.020 & 0.162 & 6.4 & 4.0 & 320.0 & 99.7 & 2,000 \\
4 & 1,210 & 0.012 & 0.084 & 5.4 & 32.1 & 450.0 & 99.8 & 2,000 \\
5 & 2,940 & 0.006 & 0.030 & 1.2 & 153.2 & 200.0 & 99.5 & 2,000 \\
6 & 4,400 & 0.003 & 0.024 & 0.2 & 66.7 & 98.5 & 2,000 \\
\hline
\end{tabular}

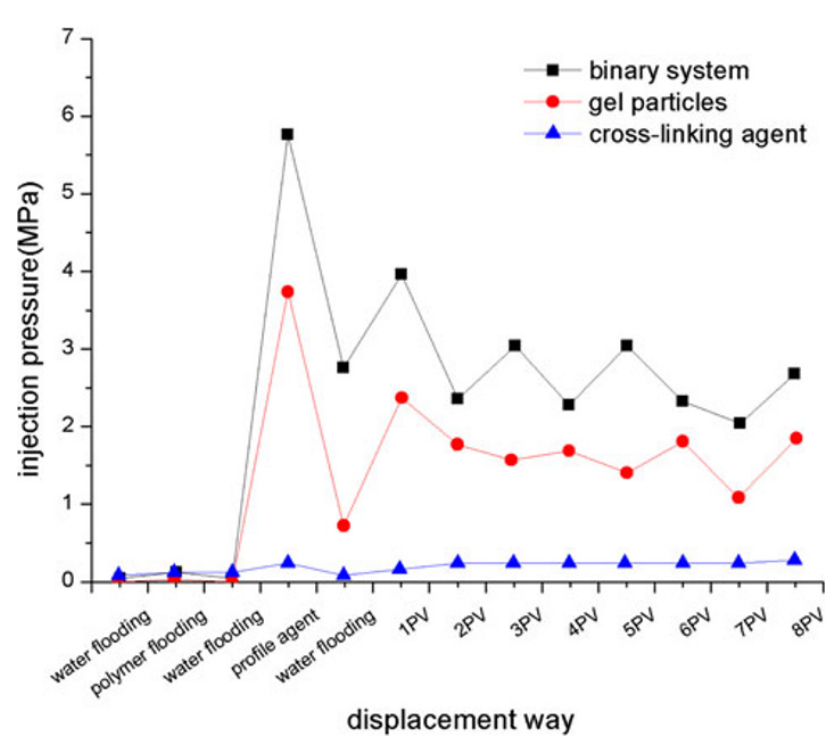

Fig. 4 Pressure variation curve of series injections

increasing as a result of electrostatic adsorption and swelling capacity after polymer flooding. The large pores are blocked by the big gel particles. The cross-linking agent cannot only form the cross-linking polymer with the residual polymer in large throats but also penetrate into small throats with low permeability which blocks the flow path to force the water flow into throats with lower permeability. The injection pressure continues to rise on account of the blocking effect.

Comparative analysis between binary injection and single injection

The binary system injection and single injection (crosslinking agent or gel particles) are separately conducted on the cores with the same permeability $(1,200 \mathrm{mD})$. The injection pressure variation curve is shown as in Fig. 4.

It can be seen from the figure that the injection pressure is nearly the same at the water and polymer flooding stage for the three injection methods. However, the injection pressure starts to show a great difference at the sequent water flooding stage for the three injection methods. The breakthrough pressure of sequent water flooding after

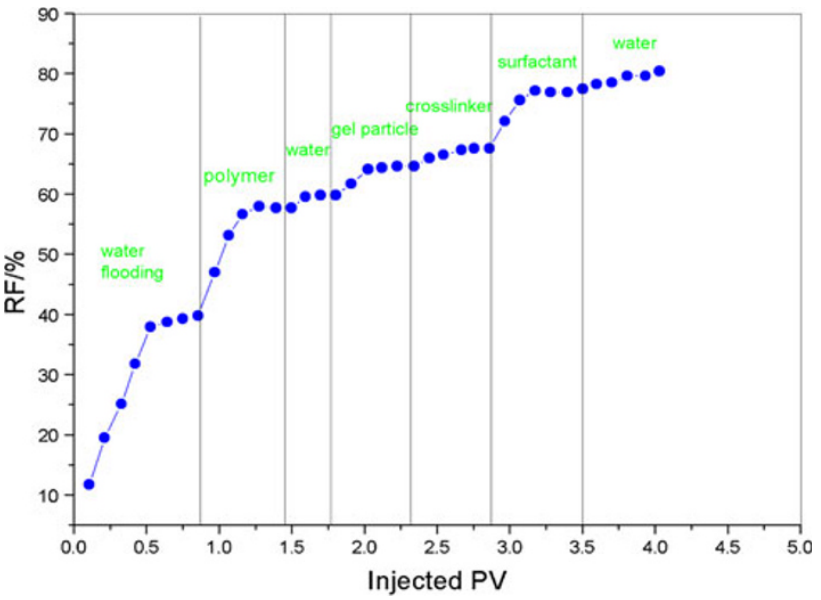

Fig. 5 Degree of reserve recovery of multiple system injection

cross-linking agent injection is relatively low and finally maintains a stable low pressure. The breakthrough of sequent water flooding after gel particles injection increases and shows some fluctuation. The breakthrough pressure of sequent water flooding after binary system injection is high. Both the fluctuation range and fluctuation extent are higher than single injection situation.

The results show that the binary system injection is better than single injection. The binary system can block the medium-high throats using the residual polymer preferably. The blocking effect can change the sequent water displacement path to improve the water flood sweep efficiency.

The multiple injection system profile control experiment

The recovery factor variation curve (Fig. 5) and recovery factor table (Table 4) can be obtained by the relationship between recover extent and injection volume factor at different injection stage of the multiple injection system core flooding experiment.

It can be seen from Table 4 and Fig. 5 that the recovery factors of water flooding and polymer flooding are limited 
Table 4 Recovery correlation table of multiple system injection

\begin{tabular}{llllllllll}
\hline Core size $(\mathrm{cm})$ & $\begin{array}{l}\text { Layer perm. } \\
\text { ratio }\end{array}$ & $\begin{array}{l}\text { Water } \\
(\%)\end{array}$ & $\begin{array}{l}\text { Polymer } \\
(\%)\end{array}$ & $\begin{array}{l}\text { Water } \\
(\%)\end{array}$ & $\begin{array}{l}\text { Gel particle } \\
(\%)\end{array}$ & $\begin{array}{l}\text { Cross-linker } \\
(\%)\end{array}$ & $\begin{array}{l}\text { Surfactant } \\
(\%)\end{array}$ & $\begin{array}{l}\text { Water } \\
(\%)\end{array}$ & $\begin{array}{l}\text { Multiple system } \\
(\%)\end{array}$ \\
\hline $30 \times 4.50 \times 4.50$ & $1: 2.9: 10.5$ & 39.65 & 18.38 & 1.93 & 4.64 & 3.48 & 9.19 & 3.58 & 22.82 \\
\hline
\end{tabular}

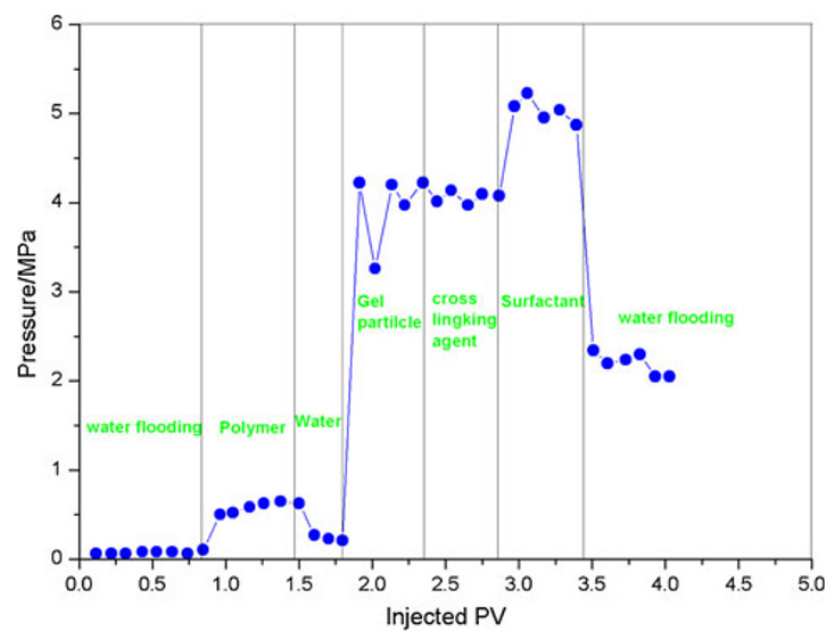

Fig. 6 Pressure variation curve of multiple system injection

and the recovery factors increase in various degrees at the cationic gel particle, cross-linking agent and surfactant injection stages.

The injection pressure variation curve is shown as in Fig. 6. The injection pressure at water and polymer flooding stage is relatively low. The injection pressure rises up to $4 \mathrm{MPa}$ after the gel particle injection and a $1.5-\mathrm{MPa}$ pressure fluctuation phenomenon comes out. The pressure continues to stay the same extent as the cross-linking agent is injected. The surfactant injection raises the injection pressure further and the injection pressure of the sequent water flooding basically stays at about $2 \mathrm{MPa}$, which is largely improved comparing with the water and polymer flooding.

In consideration of the actual construction requirements and the pumping-in equipment safety, the pumping-in pressure is usually not too high. In comparison with the binary injection system (gel particle/cross-linking agent), the sequent water flooding injection pressure of multiple injection system decreased significantly. Due to the low injection pressure and good profile control effect, the multiple injection system has pretty good practical application value.

Multiple injection system, which is used to conduct profile control in heterogeneous formations, cannot only make use of the middle-low permeability formations but also increase the pressure of the following water flooding. The multiple injection system can enhance oil recovery to a great extent.

\section{Conclusion}

1. The cationic gel particles have been screened to be used in this study; different cross-linking concentrations have different pressurization and production increasing effect, and the higher the cross-linking concentration is, the better the production performance is; the surfactant has a good depressurization and production enhanced effect and the $0.2 \%$ is the best concentration.

2. The profile control effect of binary injection system is better than that of single injection. The binary system can block the medium-high throats by using the residual polymer preferably. The blocking effect can change the sequent water displacement path to improve the water flood sweep efficiency.

3. The multiple injection system can enhance oil recovery to a great extent. The multiple injection system can not only make use of the middle-low permeability formations but also increase the pressure of the following water flooding.

Acknowledgments This work was sponsored by the National 863 Program (Grant No. 2007AA06Z00) for research on enhanced oil recovery technology after polymer flooding, the National Natural Science Foundation (Grant No. 40974056) on the particle gel profile control technology.

Open Access This article is distributed under the terms of the Creative Commons Attribution License which permits any use, distribution, and reproduction in any medium, provided the original author(s) and the source are credited.

\section{References}

Abdo MK, Chung HS (1984) Field experience with floodwater diversion by complexed biopolymers. SPE 12642

Baojun B, Liangxiong L, Yuzhang L, He L, Zhongguo W, Chunmei Y (2007a) Preformed particle gel for conformance control: factors affecting its properties and applications. SPE 89389

Baojun B, Yuzhang L, Coste JP, Liangxiong L (2007b) Preformed particle gel for conformance control: transport mechanism through porous media. SPE 89468

Coste JP, Liu Y, Bai B, Li Y, Shen P, Wang Z, Zhu G (2000) In-depth fluid diversion by pre-gelled particles: laboratory study and pilot testing. SPE 59362

Dai C, You Q, Wang Y, Zhao F, Patrick S (2010) Research on reutilization technology of residual polymer in formation after polymer flooding. SPE 129227 
Davison P, Mentzer E (1982) Polymer flooding in North Sea Reservoirs. SPE 9300

Dejun X, Hui C (2002) Crosslinked polymer flood for further enhancing oil recovery after polymer flood performed. Oilfield Chem 19(3):265-267

Dong HZ, Fang SF, Wang DM, Wang JY, Liu Z, Hong WH (2008) Review of practical experience and management by polymer flooding at Daqing. SPE 114342

Gogarty WB (1978) Micellar/polymer flooding-an overview. JPT pp 1089-1101

Jiecheng C, Jianguang W, Kaoping S, Han P (2010) Study on remaining oil distribution after polymer flooding. SPE 133808

Jishui S, Yiqiang L (2002) Oil-displacing efficiency of polymer flood and successive permeability control and polymer flood determined on three-dimensional heterogeneous physical models. Oil Field Chem 19(2):162-167

Kaminsky RD, Wattenbarger RC, Szafranski RC, Coutee AS (2007) Guidelines for polymer flooding evaluation and development. IPTC 11200

Ming H, Wentao X, Jian Z, Wei J, and Fujie S (2006) Application of EOR technology by means of polymer flooding in Bohai Oilfields. SPE 104432

Monika S, Gabriela A-J, Stefan B, Paul B, Christian S, Gregor B (2011) Sustainable surfactants in enhanced oil recovery. SPE 145039

Needham RB, Doe PH (1987) Polymer flooding review. J Petroleum Technol pp 1503-1507

Shaodong K, Xiangguo L (2002) In-depth plugging with alternative injection of cation and anion polymer and its pilot test. J Daqing Petroleum Inst 26(3):36-40
Stoll WM, Al-Shureqi H., Finol J, Al-Harthy SAA, Oyemade S, A. de Kruijf, J. van Wunnik, Arkesteijn F, Bouwmeester R, Faber MJ (2010) Alkaline-surfactant-polymer flood: from the laboratory to the field. SPE 129164

Ting X, Xiusheng L (2004) Parallel-column experiments for enhancing oil recovery after polymer flooding. Petroleum Explor Dev 31(6): 136-140

Wang D, Cheng J, Wu J, Wang Y (2002) Producing by polymer flooding more than 300 million barrels of oil, what experiences have been learnt? SPE 77872

Wang Y, Liu H (2004) How 50 million tons annual production has been maintained for 27 years in Daqing oilfield—a successful story. SPE 87048

Wei Z, Jian Z, Ming H, Wentao X, Guozhi F, and Wei J (2007) Application of hy-drophobically associating water-soluble polymer for polymer flooding in China offshore heavyoilfield. IPTC 11635

Wei Z, Jian Z, Guozhi F, Wei J, Fujie S, Shouwei Z, and Yuzhang L (2008) Keytechnologies of polymer flooding in offshore oilfield of Bohai Bay. SPE 115240

XiaoQuan W, Xijing F (2004) Water drive experiment of lowpermeability reservoir using compound surfactant. J Xi'an Shiyou Univ (Natural Science Edition) 19(5):31-32

Yefei W, Wei X, Cui J, Long H, Qing Y, Fulin Z (2006) Research on technology of formation polymer flocculation residue recycle after polymer flooding. Petroleum Geol Oilfield Dev Daqing 25(3):145-148 